\title{
The Oka-Weil theorem in topological vector spaces
}

\author{
by Bui DaC TAC (Hanoi)
}

\begin{abstract}
It is shown that a sequentially complete topological vector space $X$ with a compact Schauder basis has WSPAP (see Definition 2) if and only if $X$ has a pseudohomogeneous norm bounded on every compact subset of $X$.
\end{abstract}

The problem of approximation of holomorphic functions by polynomials in Banach spaces has been investigated by P. L. Noverraz [6], R. Aron and M. Schottenloher [1]. In 1973 C. Matyszczyk [5] generalized the results of these authors to the Fréchet space case. He showed that a Fréchet space with BAP has SPAP if and only if it has a continuous norm. In this note, we study the approximation of holomorphic functions by polynomials in topological vector spaces. In order to obtain the main results (Theorem 3 and 4) some notions for a topological vector space $X$ should be introduced.

Definition 1. We say that a sequence of operators $A_{n}: X \rightarrow Y(n=$ $1,2, \ldots)$ converges almost uniformly on an open set $Q$ in $X$ to an operator $A: X \rightarrow Y$ if $A_{n}(x) \rightarrow A(x)$ uniformly on every compact subset $K$ of $Q$.

Definition 2. We say that $X$ has the bounded approximation property, shortly BAP (resp. compact approximation property, shortly CAP) if there exists a sequence of finite-dimensional operators pointwise (resp. almost uniformly) convergent to the identity operator on $X$.

Moreover, we say that $X$ has a compact Schauder basis if $X$ has a Schauder basis $\left\{e_{j}\right\}$ such that $\left\{S_{n}(x)=\sum_{j=1}^{n} e_{j}^{*}(x) e_{j}\right\}$ converges almost uniformly to the identity operator on $X$.

Note that if $X$ is either a complete metric vector space or a barrelled locally convex space with BAP, then $X$ has CAP.

DEFINITION 3. $X$ is said to have the strong polynomial approximation property, shortly SPAP, if for every open polynomially convex subset $Q$ of 
$X$ and for every holomorphic function $f$ on $Q$, there exists a sequence of polynomials almost uniformly convergent to $f$ on $Q$.

In the case where the above property holds for open polynomially convex subsets $Q$ of $X$ of the form $Q=\bigcup_{n=1}^{\infty}$ Int $F_{n}$, where the $F_{n}$ are closed subsets of $X$ contained in $Q, X$ is said to have WSPAP.

Proposition 4. Let $X$ be a topological vector space with a compact Schauder basis. If $X$ has WSPAP, then $X$ has a norm bounded on every compact subset of $X$.

Proof. Let $\left\{e_{j}\right\}$ be a compact Schauder basis in $X$.

a) We first show that there exists a sequence $\left\{\lambda_{j}\right\} \in \mathbb{C}^{\infty}$ such that $\lambda_{j_{k}} e_{j_{k}} \nrightarrow 0$ for any subsequence $\left\{\lambda_{j_{k}}\right\}$ of $\left\{\lambda_{j}\right\}$. Assume that $D$ is an open polynomially convex set in $\mathbb{C}$ consisting of infinitely many connected components, $D=\bigcup_{j=1}^{\infty} D_{j}$, with $0 \in D$. Put

$$
G=\bigcup_{j=2}^{\infty} D_{j} e_{1}+M,
$$

where $D_{j} e_{1}=\left\{\lambda e_{1}: \lambda \in D_{j}\right\}$ and $M={\overline{\operatorname{span}\left\{e_{j}\right\}_{j \geq 2}}}_{j}$. On $G$, consider the holomorphic function $f$ given by

$$
f(z)=e_{j}^{*}(z) \quad \text { for } z \in D_{j} e_{1}+M .
$$

By hypothesis, there is a sequence of polynomials $\left\{P_{n}\right\}$, almost uniformly convergent to $f$ on $G$. For each $j \in \mathbb{N}$, consider the restriction $P_{n} \mid D_{j} e_{1}+$ $\mathbb{C} e_{j}$. Since on every compact subset of $D_{j} e_{1}+\mathbb{C} e_{j}$, this sequence converges uniformly to $e_{j}^{*}(z)=z_{j}$, where $z=z_{1} e_{1}+z_{j} e_{j}$, there exists $n_{j}$ such that $P_{n_{j}}$ depends on $z_{j}$. Thus there exists $z_{1}^{j} \in \mathbb{C}$ such that $\left|z_{1}^{j}\right|<1 / j$ and $P_{n_{j}}\left(z_{1}^{j}, z_{j}\right)$ depends on $z_{j}$. Therefore, there exists $\lambda_{j} \in \mathbb{C}$ such that $\left|P_{n_{j}}\left(z_{1}^{j}, \lambda_{j}\right)\right|>j$. We claim that $\left\{\lambda_{j}\right\}_{j=2}^{\infty}$ is the desired sequence. Indeed, assume that there exists a subsequence $\left\{\lambda_{j_{k}}\right\}$ of $\left\{\lambda_{j}\right\}$ such that $\lambda_{j_{k}} e_{j_{k}} \rightarrow 0$. Consider the compact set in $G$ given by

$$
K=\left\{z_{1}^{j_{k}} e_{1}+\lambda_{j_{k}} e_{j_{k}}, 0\right\} .
$$

Since there exists $l \geq 2$ such that $0 \in D_{l} e_{1}+M$, for $k$ sufficiently large we have

$$
z_{1}^{j_{k}} e_{1}+\lambda_{j_{k}} e_{j_{k}} \in D_{l} e_{1}+M
$$

Hence

for $j_{k}>l$ and

$$
f\left(z_{1}^{j_{k}} e_{1}+\lambda_{j_{k}} e_{j_{k}}\right)=e_{l}^{*}\left(z_{1}^{j_{k}} e_{1}+\lambda_{j_{k}} e_{j_{k}}\right)=0
$$

$$
\begin{aligned}
\left\|P_{n_{j_{k}}}-f\right\|_{K} \geq\left|P_{n_{j_{k}}}\left(z_{1}^{j_{k}} e_{1}+\lambda_{j_{k}} e_{j_{k}}\right)-f\left(z_{1}^{j_{k}} e_{1}+\lambda_{j_{k}} e_{j_{k}}\right)\right| & \geq j_{k} \\
& \text { for } j_{k}>1 .
\end{aligned}
$$


Thus $\left\|P_{n_{j_{k}}}-f\right\|_{K} \nrightarrow 0$. This contradicts the almost uniform convergence of $\left\{P_{n}\right\}$ to $f$.

b) Since $\left\{e_{j}\right\}$ is a Schauder basis of $X$, we have

$$
0=\lim _{j \rightarrow \infty} e_{j}^{*}(x) e_{j}=\lim _{j \rightarrow \infty}\left(e_{j}^{*}(x) / \lambda_{j}\right) \lambda_{j} e_{j}
$$

for each $x \in X$. Put

$$
\rho(x)=\sup _{j \in \mathbb{N}}\left|e_{j}^{*}(x) / \lambda_{j}\right|, \quad \text { where } \lambda_{1}=1 .
$$

Then $\rho$ is a norm on $X$. Since $\left\{e_{j}\right\}$ is a compact Schauder basis, $\rho$ is bounded on every compact subset of $X$. The proposition is proved.

Definition 5. A function $\rho: X \rightarrow \mathbb{R}$ is said to be a pseudo-homogeneous seminorm of degree $p>0$ if it satisfies the following conditions:

1) $\rho(x) \geq 0, \forall x \in X$,

2) $\rho(\lambda x)=|\lambda|^{p} \rho(x), \forall x \in X$ and $\forall \lambda \in \mathbb{C}$,

3) $\rho(x+y) \leq \rho(x)+\rho(y), \forall x, y \in X$.

In the case where $\rho(x)=0$ if and only if $x=0$, this pseudo-homogeneous seminorm is said to be a pseudo-homogeneous norm.

Proposition 6. Let $X$ be a sequentially complete topological vector space with CAP. If $X$ has a pseudo-homogeneous norm bounded on every compact subset of $X$, then $X$ has WSPAP.

Proof. Let $\left\{A_{j}\right\}$ be a sequence of finite-dimensional operators almost uniformly converging to the identity operator on $X$ and let $Q$ be an open polynomially convex set in $X$ such that

$$
Q=\bigcup_{n=1}^{\infty} \operatorname{Int} F_{n}=\bigcup_{n=1}^{\infty} F_{n},
$$

where the $F_{n}$ are closed sets in $X$ and $F_{n} \subseteq F_{n+1}, \forall n \geq 1$. Put

$$
Q_{j}=\{x \in Q:\|x\|<j\} \quad \text { and } \quad K_{j}=\overline{F_{j} \cap Q_{j} \cap A_{j}(X)},
$$

where $\|\cdot\|$ is a pseudo-homogeneous norm bounded on every compact subset of $X$. Then

$$
K_{j} \subseteq F_{j} \cap A_{j}(X) \subset Q \cap A_{j}(X), \quad \forall j \geq 1 .
$$

Since the topology of $A_{j}(X)$ is defined by $\|\cdot\| \mid A_{j}(X), K_{j}$ is compact in $Q \cap A_{j}(X)$. Thus by polynomial convexity of $Q \cap A_{j}(X)$, according to the Oka-Weil theorem there exists a polynomial $P_{j}$ on $A_{j}(X)$ such that

$$
\left\|P_{j}-f\right\|_{K_{j}}<1 / j .
$$

We shall prove that $\left\{P_{j}\right\}$ converges almost uniformly to $f$ on $Q$. Let $K$ be a compact subset of $Q$. Take $n_{0}$ such that $K \subset \operatorname{Int} F_{n_{0}}$. Then there exists 
a neighbourhood $V$ of zero in $X$ such that

$$
K+V \subseteq K+\bar{V} \subseteq \operatorname{Int} F_{n_{0}} .
$$

Since $A_{j}(x) \rightarrow x$ uniformly on $K$, we get

$$
A_{j}(K) \subseteq K+V \quad \text { for } j \geq j_{0} .
$$

From (1) and (2) we have

$$
A_{j}(K) \subseteq F_{n_{0}} \subseteq F_{j}, \quad \forall j \geq j_{1}=\max \left\{j_{0}, n_{0}\right\} .
$$

On the other hand, since $\bigcup_{j \geq j_{1}} A_{j}(K)$ is relatively compact and $\|\cdot\|$ is bounded on every relatively compact subset of $X$, it follows that $\bigcup_{j \geq j_{1}} A_{j}(K) \subset Q_{j_{2}}$ for some $j_{2} \geq j_{1}$. Hence

$$
A_{j}(K) \subset Q_{j}, \quad \forall j \geq j_{2} .
$$

From (3) and (4) we get

$$
A_{j}(K) \subset Q_{j} \cap F_{j} \cap A_{j}(X) \subset K_{j}, \quad \forall j \geq j_{2} .
$$

Hence

$$
\begin{aligned}
\| P_{j} A_{j} & -f\left\|_{K} \leq\right\| P_{j} A_{j}-f A_{j}\left\|_{K}+\right\| f A_{j}-f \|_{K} \\
& =\left\|P_{j}-f\right\|_{A_{j}(K)}+\left\|f A_{j}-f\right\|_{K} \leq\left\|P_{j}-f\right\|_{K_{j}}+\left\|f A_{j}-f\right\|_{K} \\
& <1 / j+\left\|f A_{j}-f\right\|_{K} \quad \text { for } j \geq j_{2} .
\end{aligned}
$$

Thus by the continuity of $f$ and since $\left\{A_{j}\right\}$ converges almost uniformly to the identity operator we infer that $\left\|P_{j} A_{j}-f\right\|_{K} \rightarrow 0$ as $j \rightarrow \infty$. The proposition is proved.

From Propositions 4 and 5 we get the following

THEOREM 7. Let $X$ be a sequentially compete vector space with a compact Schauder basis. Then $X$ has WSPAP if and only if $X$ has a pseudohomogeneous norm bounded on every compact subset of $X$.

We now consider SPAP for the class of pseudo-homogeneous topological vector spaces.

Definition 8. A topological vector space $X$ is said to be pseudohomogeneous if its topology can be defined by a family of pseudo-homogeneous seminorms.

In the case where the family of pseudo-homogeneous seminorms can be chosen countable and $X$ is complete, $X$ is said to be a pseudo-homogeneous Fréchet space.

Denote by $P(X)$ the family of all pseudo-homogeneous continuous seminorms on $X$. For each $p \in P(X)$, put

$$
U_{p}=\{x \in X: p(x) \leq 1\} .
$$


It is easy to see that

$$
p(x)=\inf \left\{\lambda^{\rho_{p}}>0: x / \lambda \in U_{p}\right\},
$$

where $\rho_{p}$ is the homogeneous degree of $p$.

We note that if $U_{p} \subseteq U_{q}$, then $\operatorname{Ker} p \subseteq \operatorname{Ker} q$, and if $p\left(x_{\alpha}\right) \rightarrow 0$, then $q\left(x_{\alpha}\right) \rightarrow 0$. Thus we can define a continuous linear map $\omega(p, q): X \widehat{X} \operatorname{Ker} p \rightarrow$ $X \widehat{K}$ er $q$. Obviously $\widehat{X}=\lim \{X \widehat{/ K} \operatorname{er} p: p \in P(X)\}$.

Theorem 9. Let $X$ be a pseudo-homogeneous Fréchet space and let $\tau$ be a pseudo-homogeneous continuous topology on $X$ such that every $\tau$-compact set is compact in $X$. Then the following properties are equivalent:

(i) every subspace of $X$ with BAP has SPAP,

(ii) there exists a pseudo-homogeneous continuous norm on $X$,

(iii) $X$ does not contain a subspace isomorphic to $\mathbb{C}^{\infty}$,

(iv) $(X, \tau)$ does not contain a subspace isomorphic to $\mathbb{C}^{\infty}$,

(v) every subspace of $(X, \tau)$ with BAP has WSPAP,

(vi) $(X, \tau)$ has a pseudo-homogeneous norm bounded on every compact subset of $X$.

To prove the theorem, we first prove the following

Proposition 10. Let $X$ be a pseudo-homogeneous Fréchet space. Then the following properties are equivalent:

(i) every subspace of $X$ with $B A P$ has $S P A P$,

(ii) there exists a pseudo-homogeneous continuous norm on $X$,

(iii) $X$ does not contain a subspace isomorphic to $\mathbb{C}^{\infty}$.

Proof. (i) $\Rightarrow$ (iii) is an immediate consequence of Proposition 4.

(iii) $\Rightarrow$ (ii). Let $\left\{p_{n}\right\}$ be an increasing sequence of pseudo-homogeneous seminorms defining the topology of $X$. If $X$ does not have a pseudohomogeneous continuous norm, then $\operatorname{dim} \operatorname{Ker} p_{n}=\infty \forall n \geq 1$. Since $\operatorname{Ker} p_{n+1} \subseteq \operatorname{Ker} p_{n}, \forall n \geq 1$, we can choose $e_{1} \in \operatorname{Ker} p_{1}$ with $p_{2}\left(e_{1}\right) \neq 0$. Since $\operatorname{dim} \operatorname{Ker} p_{2}=\infty$ and $\operatorname{Ker} p_{3} \subseteq \operatorname{Ker} p_{2}$, we find $e_{2} \in \operatorname{Ker} p_{2}$ such that $\left\{e_{1}, e_{2}\right\}$ are linearly independent and $p_{3}\left(e_{2}\right) \neq 0$. Continuing this process, we get a linearly independent sequence $\left\{e_{n}\right\}$ such that $e_{n} \in \operatorname{Ker} p_{n}, \forall n \geq 1$ and $p_{n}\left(e_{m}\right)=0$ for $m>n$. Put $X_{0}=\overline{\operatorname{span}\left\{e_{n}\right\}}$. Then $\operatorname{dim} X_{0} / \operatorname{Ker} p_{n}<\infty$, $\forall n \geq 1$. Thus $X_{0}=\varliminf X_{0} / \operatorname{Ker} p_{n} \cong \mathbb{C}^{\infty}$. This contradicts (iii).

(ii) $\Rightarrow$ (i) is an immediate consequence of Proposition 6 .

Proof of Theorem 9. We shall prove that $(\mathrm{i}) \Rightarrow(\mathrm{vi}) \Rightarrow(\mathrm{v}) \Rightarrow(\mathrm{iv})$ $\Rightarrow($ iii $) \Rightarrow($ ii $) \Rightarrow($ i $)$.

(i) $\Rightarrow($ vi). By Proposition 10 , we have (i) $\Rightarrow($ ii), and (ii) $\Rightarrow(v i)$ is trivial. Hence $(\mathrm{i}) \Rightarrow(\mathrm{vi})$. 
$(\mathrm{vi}) \Rightarrow(\mathrm{v})$ is an immediate consequence of Proposition 6 , and $(\mathrm{v}) \Rightarrow$ (iv) follows from Proposition 4. We now prove that (iv) $\Rightarrow$ (iii). Let $X$ contain a subspace $X_{0}$ isomorphic to $\mathbb{C}^{\infty}$. Since $\operatorname{dim} X_{0} / \operatorname{Ker} p<\infty$ for every $p \in$ $P(X, \tau)$ it follows that $\left(X_{0}, \tau\right)$ is a locally convex space. By a result of Martineau [4], we have $\left(X_{0}, \tau\right) \cong X_{0}=\mathbb{C}^{\infty}$.

Finally, the implications (iii) $\Rightarrow$ (ii) $\Rightarrow$ (i) follow from Proposition 10 .

Corollary 11 (Theorem 2.12 of [5]). If $X$ is a Fréchet space with BAP, then the following properties are equivalent:

(i) $X$ has SPAP,

(ii) there is a continuous norm on $X$,

(iii) $X$ contains no subspace isomorphic to $\mathbb{C}^{\infty}$.

EXAMPLES 12. 1. The following example shows that there is a locally convex space with WSPAP which does not have a continuous norm.

Denote by $(C[0,1], \tau)$ the space of all continuous functions on $[0,1]$ equipped with the topology $\tau$ defined by uniform convergence on all convergent sequences of $[0,1]$ and all seminorms defined by $\left\{e_{j}^{*}\right\}$, where $\left\{e_{j}\right\}$ is the Schauder basis in $C[0,1]$. Then $(C[0,1], \tau)$ has the following properties:

a) $(C[0,1], \tau)$ is sequentially complete with a compact Schauder basis $\left\{e_{j}\right\}$. This property follows from the fact that every convergent sequence in $(C[0,1], \tau)$ is convergent in $C[0,1]$.

b) Every $\tau$-compact subset is compact in $C[0,1]$.

c) $(C[0,1], \tau)$ does not have a continuous norm. Indeed, let $p$ be a continuous norm on $(C[0,1], \tau)$. Then there exists a sequence $\left\{t_{k}\right\}$ convergent in $[0,1]$ and $n \in N$ such that for some constant $C>0$ we have

$$
p(f) \leq C \max \left\{\sup _{k}\left|f\left(t_{k}\right)\right|, \max _{1 \leq j \leq n}\left|e_{j}^{*}(f)\right|\right\}
$$

for every $f \in C[0,1]$. Obviously this is impossible.

d) $(C[0,1], \tau)$ does not contain a subspace isomorphic to $\mathbb{C}^{\infty}$. Indeed, suppose $E$ is such a subspace. Consider the identity map $(E,\|\cdot\| \mid E) \rightarrow$ $(E, \tau \mid E)$, where $\|f\|=\sup \{|f(t)|: t \in[0,1]\}$. Since $E$ is closed in $C[0,1]$ and $(E,\|\cdot\| E)$ is a Banach space, by the open mapping theorem we get $(E,\|\cdot\| \mid E) \cong(E, \tau \mid E) \cong \mathbb{C}^{\infty}$. This is impossible.

From a), b) and from Theorem 9 it follows that $(C[0,1], \tau)$ has WSPAP. On the other hand, by $\mathrm{c}),(C[0,1], \tau)$ does not have a continuous norm.

2. Now we consider a class of spaces in which every closed ball is polynomially convex.

a) Let $X$ be a topological vector space with the Grothendieck approximation property and let $\rho$ be a continuous translation invariant metric on $X$. 
If $\rho(x, 0)$ is plurisubharmonic on $X$, then for every $x \in X$ and $r>0$, the closed ball

$$
S(x, r)=\{y \in X: \rho(x, y) \leq r\}
$$

is polynomially convex.

Indeed, let $z \notin S(x, r)$. Then there is $k$ such that $A_{k}(z) \notin S(x, r)$, where $\left\{A_{j}\right\}$ is the sequence of Grothendieck's approximation. Since $S(x, r) \cap A_{k}(X)$ is polynomially convex, there exists a polynomial $P$ on $A_{k}(X)$ such that

$$
\left|P\left(A_{k}(z)\right)\right|>1 \text { and }\|P\|_{S(x, r) \cap A_{k}(X)} \leq 1 .
$$

Put $\widetilde{P}=P A_{k}$. Then $\widetilde{P}$ is a polynomial on $X$ such that

$$
|\widetilde{P}(z)|>1 \text { and }\|\widetilde{P}\|_{S(x, r)} \leq 1
$$

b) Consider the space $L^{p}=L^{p}(X, \mu), 0<p<1$, with the metric

$$
\rho(x, y)=\int_{X}|x(t)-y(t)|^{p} d \mu \quad \text { for } x, y \in L^{p} .
$$

Then $\rho(x, 0)$ is plurisubharmonic on $L^{p}$.

Indeed, for every complex line in $L^{p}$

$$
L(\xi)=x+\xi y, \quad \xi \in \mathbb{C}, \text { where }(x, y) \in L^{p} \times L^{p} \backslash\{0\}
$$

put

$$
\varphi(\xi)=\int_{X}|x+\xi y|^{p} d \mu .
$$

We first prove that if $x, y$ are simple functions, then $\varphi(\xi)$ is subharmonic on $\mathbb{C}$. Let $x=\sum_{i=1}^{n} a_{i} \chi_{A_{i}}$ and $y=\sum_{j=1}^{m} b_{j} \chi_{B_{j}}$ where $\chi_{A_{i}}$ and $\chi_{B_{j}}$ are the characteristic functions of $A_{i}$ and $B_{j}$ respectively. Then we have

$$
\begin{aligned}
\varphi(\xi) & =\int_{X}\left|\sum_{i=1}^{n} a_{i} \chi_{A_{i}}+\xi \sum_{j=1}^{m} b_{j} \chi_{B_{j}}\right|^{p} d \mu \\
& =\sum_{i, j} \int_{A_{i} \cap B_{j}}\left|a_{i} \chi_{A_{i}}+\xi b_{j} \chi_{B_{j}}\right|^{p} d \mu=\sum_{i, j}\left|a_{i}+\xi b_{j}\right|^{p} \mu\left(A_{i} \cap B_{j}\right) \\
& =\sum_{i, j}\left|a_{i}+\xi b_{j}\right|^{p} \alpha_{i j}=\sum_{i, j}\left[\left(a_{i}+\xi b_{j}\right)\left(\bar{a}_{i}+\overline{\xi b_{j}}\right)\right]^{p / 2} \alpha_{i j},
\end{aligned}
$$

where $\alpha_{i j}=\mu\left(A_{i} \cap B_{j}\right)$. Hence

$$
\begin{aligned}
\partial \varphi / \partial \xi=\sum_{i, j} & (p / 2) \alpha_{i j}\left[\left(a_{i}+\xi b_{j}\right)\left(\bar{a}_{i}+\overline{\xi b}_{j}\right)\right]^{p / 2-1} b_{j}\left(\bar{a}_{i}+\overline{\xi b}_{j}\right) \\
\partial^{2} \varphi / \partial \bar{\xi} \partial \xi= & \sum_{i, j}(p / 2)(p / 2-1) \alpha_{i j}\left[\left(a_{i}+\xi b_{j}\right)\left(\bar{a}_{i}+\overline{\xi b}_{j}\right)\right]^{p / 2-2} b_{j} \bar{b}_{j}\left(a_{i}+\xi b_{j}\right) \\
& \times\left(\bar{a}_{i}+\overline{\xi b}_{j}\right)+(p / 2) \alpha_{i j} b_{j} \bar{b}_{j}\left[\left(a_{i}+\xi b_{j}\right)\left(\bar{a}_{i}+\overline{\xi b}_{j}\right)\right]^{p / 2-1}
\end{aligned}
$$




$$
=(p / 4) \sum_{i, j} \alpha_{i j}\left|b_{j}\right|^{2}\left[\left(a_{i}+\xi b_{j}\right)\left(\bar{a}_{i}+\overline{\xi b}_{j}\right)\right]^{p / 2-1} \geq 0
$$

for $\xi \in \mathbb{C} \backslash \bigcup_{i, j}\left\{\xi: a_{i}+\xi b_{j}=0\right\}$. From this and from the continuity of $\varphi$ on $\mathbb{C}$ it follows that $\varphi$ is subharmonic on $\mathbb{C}$.

Let now $(x, y) \in L^{p} \times L^{p} \backslash\{0\}$. Then there exists two sequences of simple functions $\left\{x_{n}\right\}$ and $\left\{y_{n}\right\}$ such that $\int_{X}\left|x-x_{n}\right|^{p} d \mu \rightarrow 0$ and $\int_{X}\left|y_{n}-y\right|^{p} d \mu$ $\rightarrow 0$. Put

$$
\varphi(\xi)=\int_{X}|x-\xi y|^{p} d \mu, \quad \varphi_{n}(\xi)=\int_{X}\left|x_{n}-\xi y_{n}\right|^{p} d \mu .
$$

Then

$$
\left|\varphi(\xi)-\varphi_{n}(\xi)\right| \leq \int_{X}\left|x-x_{n}\right|^{p} d \mu+|\xi|^{p} \int_{X}\left|y-y_{n}\right|^{p} d \mu \rightarrow 0
$$

uniformly on every compact subset of $\mathbb{C}$. Thus $\varphi(\xi)$ is subharmonic on $\mathbb{C}$.

From the first example it follows that if $L^{p}(X, \mu)$ has the Grothendieck approximation, then every closed ball in $L^{p}(X, \mu)$ is polynomially convex.

In the case where $L^{p}(X, \mu)$ does not have the Grothendieck approximation, no closed ball in $S(x, r)$ can be polynomially convex. For example, consider the space $L^{p}[0,1], 0<p<1$. It is known that $\left(L^{p}[0,1]\right)^{\prime}=\{0\}$. This implies that every polynomial on $L^{p}[0,1]$ is constant. Thus no closed ball in $L^{p}[0,1]$ is polynomially convex.

\section{References}

[1] R. M. Aron and M. Schottenloher, Compact holomorphic mappings on Banach spaces and the approximation property, J. Funct. Anal. 21 (1976), 7-30.

[2] A. Bayoumi, The Levi problem and the radius of convergence of holomorphic functions on metric vector spaces, in: Lecture Notes in Math. 834, Springer, 1981, 9-32.

[3] - Bounding subsets of some metric vector spaces, Ark. Mat. 18 (1980), 13-17.

[4] A. Martineau, Sur une propriété caractéristique d'un produit de droites, Arch. Math. (Basel) 11 (1960), 423-426.

[5] C. Matyszczyk, Approximation of analytic and continuous mappings by polynomials in Fréchet spaces, Studia Math. 60 (1977), 223-238.

[6] P. L. Noverraz, Pseudo-convexité, Convexité Polynomiale et Domaines d'Holomorphie en Dimension Infinie, North-Holland Math. Stud. 3, Amsterdam 1973.

DEPARTMENT OF MATHEMATICS

PEDAGOGICAL INSTITUTE 1

HANOI, VIETNAM 